\title{
Inter
}

Art actuel

\section{Tenir compte du contexte : le performatif}

\section{Richard Martel}

Numéro 105, printemps 2010

Fragments d'art actif

URI : https://id.erudit.org/iderudit/62647ac

Aller au sommaire du numéro

Éditeur(s)

Les Éditions Intervention

ISSN

0825-8708 (imprimé)

1923-2764 (numérique)

Découvrir la revue

Citer cet article

Martel, R. (2010). Tenir compte du contexte : le performatif. Inter, (105), 4-5. d'utilisation que vous pouvez consulter en ligne.

https://apropos.erudit.org/fr/usagers/politique-dutilisation/ 


\section{Tenir compte du contexte : le performatif}

- RICHARD MARTEL

L'art performance - ou art action - aura réellement transformé les pratiques d'art vivant, et dans plusieurs disciplines. C'est un fait maintenant vérifiable, et les pratiques en danse, en théâtre, en photo, en vidéo, en audio, en multimédia et autres démontrent l'importance du fait performatif dans l'ensemble des données du spectaculaire, plus ou moins diffus, concentré ou même aggloméré.

L'univers performatif a donc contribué à l'émancipation des pratiques diverses de l'expressivité tout autant plastique que sonore ou environnementale. En optimisant la dynamique envers ce qui est statique, c'est une grande partie de l'édifice artistique et culturel qui doit être pris en considération dans la pluralité des systèmes esthétiques. II y a un mélange qui implique, qui lie, au sens d'assemblages, d'interconnexions potentielles, et le contexte reçoit une densité variable par des énergies qui circulent, qui sont dans des états d'interdépendance. II n'y a plus de relationnisme - dans l'atelier traditionnel. II s'agit donc de prendre en considération le contexte de l'énonciation. Ce même contexte suscite des aménagements, des "stimulants", et ce, en fonction même des rapports entre les matières dans l'espace-temps qui déterminent aussi la teneur du geste ou du processus.

Même la publicité, surtout la promotion publicitaire, a orienté la communication consommatrice dans son langage : ne parlet-on pas de performances d'automobile, de banque, d'industrie... ? Certes, le performatif convient bien aux sociétés articulées sous l'angle du progrès, dans l'empire tentaculaire des dispositifs économiques ; il est clair que la performance devient un leitmotiv dans l'axe de progression du dispositif.

Retracer l'histoire de l'art action et de la performance pourrait nous amener assez loin dans le temps historique, ancien, tout autant chez les cyniques que les occultistes ou actionnistes. Et certes, cette notion d'alchimie convient bien aussi au performatif. II y a en outre une « histoire » de l'art action et de la performance, et les publications récentes sont des témoignages de gestes posés par les artistes et poètes. Le fait de présenter des exécutions langagière et gestuelle entraîne une situation à laquelle la notion de présence s'imbrique. Parce qu'il s'agit de vivre une situation demandant plus ou moins de partage, avec un public présent - et relativement à lui, selon le nombre -, il y a osmose et réciprocité, colportant l'idée de fusion, interpellant la relation. On pourrait aussi illustrer le propos par des cas, des moments historiques du développement de la discipline. Mais d'autres I'ont déjà fait.

Déjà au XIXe siècle, au cabaret Le Chat noir à Paris par exemple, il y a mixité des langages en gestation, du poétique au musical, du théâtral au philosophique. Puis, après le futurisme, le dadaïsme, les exemples sont nombreux et s'intensifient avec les années soixante : Fluxus, happening, art corporel, poésie action, etc. Le moment de l'action est aussi déterminé par le contexte de l'énonciation, et le public présent agit sur la présentation du performatif.

Mais c'est d'abord par la présence que se concrétise l'acte esthétique puisqu'il fait appel et réfère, qu'il implique des éléments d'une certaine plasticité et qui se charge d'un appareillage pulsionnel dans un désir " partageant», dans le sens d'une construction, d'un délire, d'un dégagement des tissus conventionnels : une proposition, donc, située plus du côté d'une remise en question. Par l'implication directe de la personne, l'univers artistique et poétique s'est trouvé « subjectivisé » de l'intérieur, tel un « transformationnel », une puissance dans l'acte. Il s'agit de considérer des déplacements d'énergie, de vivre directement par une situation des conditions d'activation plus ou moins déterminantes. Par le positionnement agi, l'univers de l'art action insinue la présence, mais aussi l'utilisation de données se « maximalisant » dans un temps et un espace. Or, il s'agit d'abord d'en analyser le potentiel d'affirmation.

Aussi, aucun lieu n'est neutre : il est chargé d'identités, de cultures, de zones plus ou moins codées, à partir de données plus ou moins conventionnelles. Et le fait de présenter une proposition dans un contexte public instaure le moment dans un rapport d'interdépendance : il y a nécessairement un risque dans les relations et déplacements, car cela s'ajuste, ou non, et se relativise.

La performativité aura ainsi contribué à sortir l'acte pour l'art des contextes institués par la norme, principalement avec la muséologie. II s'agit ici d'un certain nivellement. En imprimant une présence d'acte dans une situation vécue, une génération-corruption, disons, immédiate se produit ; c'est un moment qui permet la réflexion et qui sollicite un temps réflexif dénormalisé, aléatoire ; c'est un moment qui permet la diversité des approches et des significations.
Le performatif aura spécifié de revenir au corps, à la présence, à la matière corporelle dans son apport aux diverses architectures, du symbolique au réel, du conceptuel au naturel. On pourrait aussi remarquer que le développement des outils et des méthodologies, avec les machines et leurs caractéristiques, aura suscité un paradoxal retour à l'expressivité - individuelle souvent tout en offrant d'autres possibilités dans l'énonciation d'un corps politique, d'une nature dénaturant les conventions. En effet, il s'agit souvent, dans le poétique et l'artistique, de proposer des zones dites alternatives, un courant autre que délimitatif, une présence chaotique agissant dans les univers plus ou moins standardisés du culturel, et ce, en sachant fort bien que c'est de plus en plus arbitraire à l'époque de la mondialisation. Il y aurait de plus des zones géographiques performatives qui auraient plus de densité que d'autres. C'est un fait d'histoire et un moment du « civilisationnel ». Ça aussi, c'est relatif.

Chaque univers culturel comporte des caractéristiques, des styles, des rejets comme des préoccupations communs. Un univers culturel n'est pas neutre. Toutefois, il y aurait une sorte de mondialisation des conditions de matières esthétiques. Or, c'est la responsabilisation de l'auteur de dissimuler, de démontrer ou de propager de la couleur dans les univers plus ou moins standardisés de la culture, et il y a quand même de la place pour l'arbitraire, le questionnement, le relatif. L'implication de la personne est aléatoire et, tout comme le style, elle est plus ou moins personnelle, elle s'identifie tout autant au moi personnel qu'à l'activisme politique. L'univers performatif est élastique, et chaque «personnificateur » d'une proposition performative peut énoncer un univers personnel ou collectif. Le performatif est souple, les ajustements sont en fonction de l'implication, du narcissisme ou de la cause politique ; le moi est ambivalent, et l'occasion peut déterminer la structure et le développement de l'acte dans les univers troubles du culturel. Le performatif aura permis de sortir des systèmes pédagogiques et des institutions. II propose d'agir contre le déterminé d'avance. C'est un avancement dans des déterminations qui sont subjectives et qui peuvent donc se transformer par l'usage et en fonction des lieux et des circonstances.

Performances mexicaine, japonaise, polonaise, française, italienne... Les cultures 
Revenir au corps, à la présence, à la matière corporelle dans son apport aux diverses architectures, du symbolique au réel, du conceptuel au naturel.

sont aussi des conditionnements. Présenter une action dans un festival de poésie, en France, disons, est totalement différent de montrer une proposition performative dans un centre pour l'art alternatif au Mexique. Une action sera bien reçue à un endroit et mal reçue à un autre. Tout phénomène culturel ou artistique est ambivalent et contribue à une sorte d'identité, de modèle ou de conditionnement.

Performance pour les sens, pour divertir ; performance pour l'intellect, pour réfléchir ; l'univers du performatif est pluriel, polyphonique. Pour avoir présenté des activités performatives au cours des 25 dernières années, je peux rendre compte du climat culturel. C'est peut-être une constante ailleurs, mais les premières générations d'artistes de la performance semblaient - et je dis semblaient agir pour une transformation externe, au sens politique, social, tandis que les générations récentes semblent plutôt agir pour une expressivité interne, plus individuelle.

Bien que les récents systèmes performatifs relationnels, paradoxalement impliquant le public, soient un réel témoignage de la personne d'abord, dans la fabrication et la digestion de la matière performative, il s'agit toutefois, en voulant affirmer le participatif par son rapport au relationnel, de réhabiliter le pouvoir de l'auteur dans l'accomplissement des séquences artistique et poétique. Mais comment ne pas comprendre que l'éphémérité et la rapidité des moyens technologiques puissent influencer les modèles esthétiques ? Lorsqu'on a la possibilité d'avoir accès à la photo ou à la vidéo quelques minutes seulement après la présentation d'une activité, cela occasionne un rapport à la trace et au produit d'une manière assez spéciale. Auparavant, un artiste pouvait attendre près d'une à deux semaines avant de visualiser son activité.

Une dimension d'immédiateté à l'action sollicite une certaine propension à la culture d'un moi, principalement. Cela peut être pris en considération dans les motivations et l'implication idéologique entre une culture expressive du moi, qui peut virer au narcissisme, et une implication sociale, qui insiste sur une transformation à venir, ce qui explique, aussi, les écarts de générations.

Prenons un exemple simple : un étudiant en art réalise une performance dans un cours ; on en fait une captation vidéo, qui se retrouve sur YouTube... circule, se copie, «s'endisque» même. Une culture de l'immédiat totémise l'art par rapport à sa dimension d'analyse culturelle critique.

À l'ère de la globalisation, les outils technologiques, les diverses machines et les instruments de toutes catégories semblent presque être les mêmes, un peu partout. Par exemple, la vidéo, qui est maintenant un fait informatique, numérique, a entraîné une nouvelle uniformisation, presque même planétaire. Dans tous les pays ou presque, disons, développés, les mêmes outils servent aux artistes comme à « l'homme de la rue ». Il est de plus en plus probable d'assister à un phénomène de standardisation de l'expressivité, et cela peut se vérifier dans les activités performatives, même s'il ne faut pas tout généraliser.

Il faut tenir compte du contexte de l'énonciation parce qu'aucun lieu n'est neutre, mais encore que cette même situation est codée et qu'elle détermine des conventions et des usages. Tout participe donc au performatif : l'espace comme le temps, le bagage culturel, individuel comme collectif, les zones de tabous comme les habitudes, les tolérances comme les interdictions. Dans l'univers plus ou moins standardisé de la culture et des appareils pour l'incarner, il y a possibilité de librearbitre poétique et artistique. C'est à l'auteur de déterminer le degré d'actualisation de sa présence dans l'énormité des systèmes de langages présentés. L'univers du performatif est une sorte d'offrande et doit se vivre, donc, par la conscience, telle une distillation, au sens alchimique, en fonction des normes et par une responsabilisation relative aux structures ambiantes.

Richard Martel est producteur et déstabilisateur. Il écrit, performe, fait de l'installation, de la vidéo, s'occupe de diffusion, organise et dissémine l'art par l'entremise du Lieu, centre en art actuel, et de la revue Inter, art actuel. II a réalisé plus de 240 performances dans plus de 40 pays... 